\title{
Clonal Structure of Two Wild Lotus (Nelumbo nucifera Gaertn.) Populations Inferred from Amplified Fragment Length Polymorphism Fingerprints
}

\author{
Kazunari Nomura ${ }^{1}$, Tamae Harada ${ }^{1}$, Nozomi Saotome ${ }^{1}$, Minami Kubota ${ }^{1}$, Masato Katori ${ }^{2}$ \\ ${ }^{1}$ College of Bioresource Sciences, Nihon University, 1866 Kameino Fujisawa, Kanagawa, Japan \\ ${ }^{2}$ Suigo-Sawara Aquatic Botanical Garden, Ogishima, Katori, Chiba 287-0801, Japan
}

Email address:

nkazu@brs.nihon-u.ac.jp (K. Nomura)

\section{To cite this article:}

Kazunari Nomura, Tamae Harada, Nozomi Saotome, Minami Kubota, Masato Katori. Clonal Structure of Two Wild Lotus (Nelumbo nucifera Gaertn.) Populations Inferred from Amplified Fragment Length Polymorphism Fingerprints. Plant. Vol. 3, No. 1, 2015, pp. 8-14.

doi: 10.11648/j.plant.20150301.12

\begin{abstract}
The lotus Nelumbo nucifera often forms dense populations at the margins of lakes and ponds. Many aquatic plant species such as $N$. nucifera are characterized by the ability to reproduce both sexually and asexually, which can affect the genetic diversity of populations growing in different types of environments. We compared the clonal diversities of two natural lotus populations in Japan. Diversity was quantified by DNA fingerprinting of amplified fragment length polymorphisms (AFLPs) in leaves sampled across each population. The DNA fingerprints revealed different amounts of diversity in each population. In 162 samples from Lake Kasumigaura, where lotus has grown continuously for many years, 35 AFLP patterns were detected. One particular clone was distributed widely, suggesting that growth of seed-derived plants was inhibited because of the superior competitive ability of rhizomes, resulting in low genetic diversity within the population. In 214 samples from Uchi-numa Pond in Miyagi Prefecture, where the lotus population was totally destroyed by heavy rains in 1998 and is now recovering, 213 different AFLP profiles were detected. The spatial distance between samples from Uchi-numa Pond was correlated with the genetic distance. These results suggest that, in lotus, sexual reproduction is favored when rapidly varying water level damages the population, because small seedlings are better able to survive in a low-density population. In contrast, vegetative reproduction is favored when environmental conditions are stable.
\end{abstract}

Keywords: Aquatic Plants, Clonal Reproduction, Genotypic Diversity, Nelumbo nucifera

\section{Introduction}

Lotus belongs to the genus Nelumbo, which has only two species. One species, Nelumbo nucifera Gaertn., with pink or white flowers, is found in Asia and northern Australia. N. nucifera is widely distributed in Japanese eutrophic waters [1-3]. Wild $N$. nucifera often forms dense populations at the margins of lakes and ponds. Recently, dredging and shore-protection projects have caused profound changes in aquatic ecosystems. These changes include reduction in biodiversity, degradation of habitat, and acceleration of nutrient cycling and eutrophication [4].

Many aquatic plant species, including N. nucifera, are characterized by the ability to reproduce both sexually and clonally. They grow vigorously by extending rhizomes, but they can also produce seeds to generate sexual progenies. In such plants, "individuals" can be recognized at two different organizational levels: genets and ramets. A genet is composed of all tissue originating from one zygote, whereas a ramet is an independent part of a genet derived by asexual reproduction [5]. Lotus plants flower from June to August in Japan; the seeds are released in autumn and dispersed by water currents. Lotus flowers are open-pollinated by insects and wind [6], and heterozygous individual plants bear genetically segregating offspring. In contrast, rhizomes branch and extend horizontally in the soil. Many newly branched rhizomes grow from the nodes of the rhizome straps, resulting in clonal populations. Cultivated lotus often extends rhizomes vigorously to over $10 \mathrm{~m}$ per year. Terminal buds of the rhizome sprout into new rhizome straps, which grow to 20 to $30 \mathrm{~cm}$ in soil before flowering. The straps extend horizontally and many branched straps with a diameter of 10 to $15 \mathrm{~mm}$ grow out from their nodes. The primary branch stems are formed continuously from the nodes of main stem, and 
secondary branch stems are generated from the lower nodes of primary stems forming a large expanse of stem-complex with the terminal bud [7]. Clones of lotus spread quickly in horizontal space in early stage of the population whereas they make packed clumping ramets in late stage.

Han et al. [6] estimated genetic diversity of natural populations of Nelumbo nucifera based on ISSR loci, and revealed that genetic diversity was high at the species level but low within the individual study populations. Xue et al. [8] studied genetic diversity of wild lotus in Heilonjiang Province in China, and showed that the very limited genetic diversity. Little studies have been carried out to elucidate the importance of a pattern of the variations in asexual and sexual reproduction of a clonal plant, although it is critical for understanding the causes of variation of genetic diversities.

The genetic structure of each population is determined by the balance of the two reproductive modes: sexual and clonal [9]. The importance of sexual vs. clonal reproduction and its relationship to genetic variation in natural populations has been studied in several aquatic species $[10,11]$. The mode of reproduction corresponds to a remarkable difference in genotypic diversity [12]. Sexual reproduction in clonal plants is likely to bear genetic variation, whereas asexual reproduction reduces the variation. Little is known about how many clones exist in a population and how large an area does a single clone occupy. $N$. nucifera is one of ideal species for examining the variation in patterns of clonal and sexual reproduction and how environmental conditions affect the genetic diversity of the populations. The number of clones and the area of a single clone in a population would reveal the balance of sexual and clonal reproduction. We could predict the environmental factors affected on the balance, however, few studies have given a clear pattern of clonal structure in a population of $N$. nucifera.

Genetic markers are required to survey the distribution of a clone derived from a single seed in a population of clonal plants. It has become possible to study clonal structure in natural plant populations using molecular genotyping. In this study, we used AFLP fingerprinting to compare the clonal diversity of two natural populations of lotus ( $N$. nucifera) in Japan. The two locations differ in the degree to which water level varies over time. The aims of this study were to determine the genetic variation within the two populations of wild lotus which can produce offspring through clonal reproduction and seedling recruitment simultaneously and to elucidate the relationship between genetic variation and reproduction system. Our results suggested that the trade-off between sexual and clonal reproduction is influenced by environmental conditions such as water level.

\section{Materials and Methods}

\subsection{Study Plots and Sampling}

Two areas containing lotus populations were investigated (Fig. 1). The first area was located at the north side of Lake Kasumigaura in Ibaraki Prefecture, Japan $\left(36^{\circ} 07^{\prime} \mathrm{N}\right.$, $\left.140^{\circ} 22^{\prime} \mathrm{E}\right)$, an area where lotus has grown continuously for many years. A dense population of $N$. nucifera occupies the margin of the lake, surrounding artificial floating islands and extending along a shore-protecting embankment. The maximum and mean depths are $7.3 \mathrm{~m}$ and $4.0 \mathrm{~m}$, respectively in Lake Kasumigaura. The lake has 26 small inflows and one outflow. The distribution of $N$. nucifera is restricted to within a region of less than $1.5 \mathrm{~m}$ water depth. A rise of the water level after a severe typhoon caused a subsequent decrease in biomass of $N$. nucifers, however, never extinguished rhizomes totally [13]. The area of lotus growth has been decreasing due to the lakeside construction and dredging within the lake. The Lake Kasumigaura plot was divided into site A $\left(35 \times 130 \mathrm{~m}^{2}\right)$ and $\mathrm{B}\left(90 \times 40 \mathrm{~m}^{2}\right)$. Site B was about $500 \mathrm{~m}$ from site A.

The second area was located in Uchi-numa Pond, located in northern Miyagi Prefecture (38 $\left.43^{\prime} \mathrm{N}, 141^{\circ} 04^{\prime} \mathrm{E}\right)$ in Japan. In Uchi-numa Pond, the maximum and mean depths are $1.6 \mathrm{~m}$ and $0.8 \mathrm{~m}$, respectively. The pond has two inflows and no outflow. Most of regions are possible to grow for the species in the pond. In Uchi-numa Pond, heavy flooding has damaged to extinguish to lotus once in 20 to 30 years. Before 1998, a dense population of $N$. nucifera was formed; however, the population was totally destroyed by a severe flooding in 1998 . The population has been recovering for about 10 years, and a new population has recently become established. The plot size at Uchi-numa Pond was consisted of site A $\left(40 \times 270 \mathrm{~m}^{2}\right)$ and B $\left(10 \times 50 \mathrm{~m}^{2}\right)$.

The population size of Uchi-numa Pond was larger than the size of Lake Kasumigaura. The sites at each site were divided into grids $(5 \times 5 \mathrm{~m}$ sections in Lake Kasumigaura and $10 \times 10$ $\mathrm{m}$ sections in Uchi-numa Pond), and one leaf sample was collected from each section in 2006 and 2008.

\subsection{DNA Extraction}

Fresh leaf samples were used to extract total DNA. Leaves were cut into small pieces and washed with tap water followed by distilled water. Total DNA was extracted from about $1 \mathrm{~g}$ of each sample by using a modification of the method of Murray and Thompson [14]. Sample DNA was dissolved in 1/10 TE buffer (0.1 mM Tris-HCl, $0.01 \mathrm{mM}$ EDTA).

The template is used to format your paper and style the text. All margins, column widths, line spaces, and text fonts are prescribed; please do not alter them. You may note peculiarities. For example, the head margin in this template measures proportionately more than is customary. This measurement and others are deliberate, using specifications that anticipate your paper as one part of the entire publication, and not as an independent document. Please do not revise any of the current designations.

\subsection{AFLP Procedure}

The protocol used for analysis was developed by Vos et al. [15] and modified slightly here. DNA from each sample was digested with 10 units of EcoRI (New England Biolabs, Inc. Ipswich, MA, USA ) and 25 units of MseI (New England Biolabs, Inc. Ipswich, MA, USA) in buffer containing $10 \mathrm{mM}$ 
Tris-acetate $(\mathrm{pH} 7.5), 10 \mathrm{mM}$ magnesium acetate, $50 \mathrm{mM}$ potassium acetate, $5 \mathrm{mM}$ dithiothreitol, and $50 \mathrm{ng} / \mu \mathrm{L}$ bovine serum albumin in a total volume of $25 \mu \mathrm{L}$ at $37^{\circ} \mathrm{C}$ for $90 \mathrm{~min}$, with termination at $65^{\circ} \mathrm{C}$ for $20 \mathrm{~min}$. The digested solution was mixed with a ligation mixture containg 50 pmol of EcoRI and MseI adapter, 1x ligation buffer, and 28 unit T4 DNA ligase (Nippon Gene, Tokyo, Japan), and the mixture was incubated at $16^{\circ} \mathrm{C}$ overnight. After ligation, the mixture was diluted to $10 \%$ of its original concentration using TE buffer, and $1 \mu \mathrm{L}$ of that dilution was subjected to primary PCR amplification. The reaction mixture consisted of 2 pmol of the adapter primers, $0.25 \mathrm{mM}$ dNTPs, $1 \mathrm{x}$ PCR buffer, and 1 unit rTaq polymerase (Takara Bio Inc., Shiga, Japan), in a total volume of $10 \mu \mathrm{L}$. The PCR was run with a program consisting of 20 cycles of $94{ }^{\circ} \mathrm{C}$ for $30 \mathrm{~s}, 56^{\circ} \mathrm{C}$ for $30 \mathrm{~s}$, and $72{ }^{\circ} \mathrm{C}$ for 1 min. Following the primary PCR, the reaction mixture was diluted to $4 \%$ of its original concentration with TE buffer before selective amplification. The reaction was performed in the same reaction mixture used for the primary PCR. For selective PCR amplification, primers with an additional three bases, $E$-AAA $/ M$-CTC, $E$-AAG $/ M$-ACG, $E$-ACG $/ M$-ACA and $E-\mathrm{AGT} / M-\mathrm{GCG}$, were used. The first segment of the PCR program consisted of 13 cycles of $30 \mathrm{~s}$ at $94^{\circ} \mathrm{C}$, followed by 30 -s annealing temperatures that decreased from $65^{\circ} \mathrm{C}$ by 0.7 ${ }^{\circ} \mathrm{C}$ per cycle and a final min at $72{ }^{\circ} \mathrm{C}$, and the second segment consisted for 23 cycles of $94^{\circ} \mathrm{C}$ for $30 \mathrm{~s}, 56^{\circ} \mathrm{C}$ for $30 \mathrm{~s}$, and 72 ${ }^{\circ} \mathrm{C}$ for $1 \mathrm{~min}$. The amplification products were size fractionated by electrophoresis on polyacrylamide gels using 100 bp ladders as a size marker. The fragments were visualized using the Silver Stain Plus (Bio-Rad, USA) according to manufacturer's instructions. Each electrophoresis was performed two times, and only reproductive bands were used for a following estimation.

\subsection{Data Analysis}

Reproducible AFLP fragments from 194 to $1353 \mathrm{bp}$ in length were scored as "present" or "absent". Samples that showed identical AFLP band patterns were classified as coming from the same clone, whereas samples that showed different profiles were classified as being from different clones. Genetic distances between pairs of clones were calculated by the formula of Nei and Li [16]. The genetic distance matrix among clones was used to construct a dendrogram using an unweighted pair group method with arithmetic averaging (UPGMA) and the program PHYLIP version 3.69 [17]. The distances between the centers of the grid sections within a plot were used as the geographical distances between samples. Two measures of genotypic diversity were calculated in accordance with the method of Ellstrand and Roose [18]: (i) $P=G / N r$, where $G$ is the number of distinguishable genotypes and $\mathrm{Nr}$ is the total number of sampled ramets; (ii) Simpson's index of diversity corrected for finite sample size, given by $D=1-\left\{\left[\sum n_{\mathrm{i}}\left(n_{\mathrm{i}}-1\right)\right] /[\mathrm{Nr}(\mathrm{Nr}-\right.$ 1) $]\}$, where $n_{\mathrm{i}}$ is the number of individuals with the same genotype and $\mathrm{Nr}$ the number of ramets sampled [19]; and (iii) genotypic richness, $\mathrm{R}=(G-1) /(N r-1)[20]$.

\section{Results}

\subsection{Lake Kasumigaura Population}

DNA samples from 162 out of 222 leaves from Lake Kasumigaura were available for analysis and remaining 60 samples had no analytical AFLP bands. Out of 162, 29 samples had unique patterns. The remaining 133 samples fell into six groups each consisting of 2 to 122 samples with identical profiles. In total, 35 AFLP band patterns were detected in the 162 samples.

The spatial distribution of the 35 clones is illustrated in Fig. 2. One particular clone (designated "1") was found throughout sites A and B. One hundred twenty-two samples were assigned to this clone. The distance between the most distant samples in this clone was $720 \mathrm{~m}$. For the other genotype groups, samples having the same AFLP band patterns were not always close together. When the AFLP band patterns of the 35 clones were compared in pairs, total of 60.1 fragments could be detected using the 4 primer sets, and a mean of 13.1 fragments $(21.8 \%)$ were polymorphic between two clones in 35 clones (Table 1).

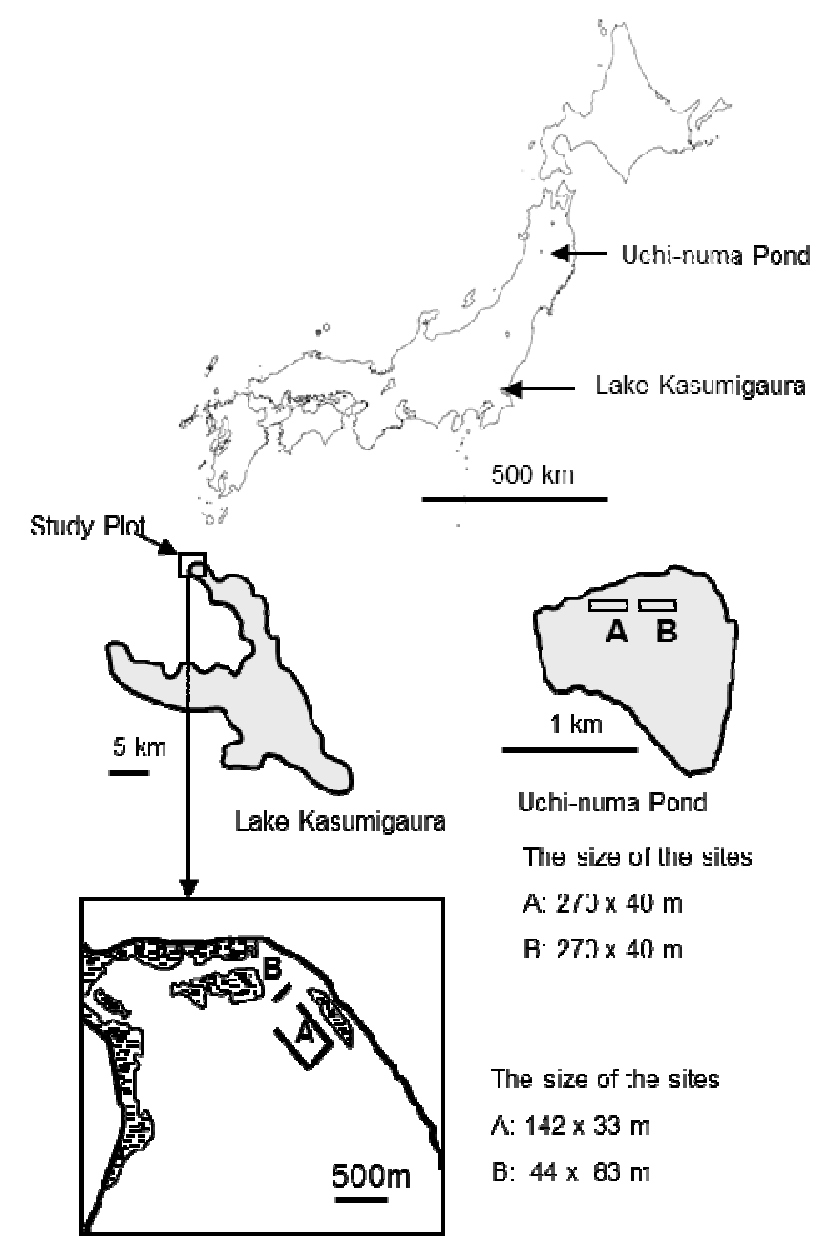

Figure 1. Location of the populations of Nelumbo nucifera sampled in this study. $A$ and $B$ indicate sites at each location. 
Table 1. Amplified fragment length polymorphisms (AFLPS) detected in clones from Lake Kasumigaura and Uchi-numa Pond

\begin{tabular}{lllll}
\hline Primer pair & $\begin{array}{l}\text { Mean number of } \\
\text { fragments per sample }\end{array}$ & $\begin{array}{l}\text { Mean number of } \\
\text { fragments per pair of } \\
\text { clones }\end{array}$ & $\begin{array}{l}\text { Mean number of } \\
\text { polymorphic fragments } \\
\text { per pair of clones }\end{array}$ & $\begin{array}{l}\text { Mean polymorphisms } \\
\text { between clones (\%) }\end{array}$ \\
\hline Lake Kasumigaura (35 clones) & & & & \\
$E-A A A / M-C T C$ & 19.0 & 21.2 & 2.4 & 20.1 \\
$E-A A G / M-A C G$ & 12.3 & 15.4 & 3.1 & 28.1 \\
$E-A C G / M-A C A$ & 13.0 & 12.1 & 3.4 & 36.8 \\
$E-A G T / M-G C G$ & 9.0 & 11.4 & 4.2 & 21.8 \\
Total & 53.3 & 60.1 & 13.1 & 85.4 \\
Uchi-numa Pond (213 clones) & & & 17.2 & 75.0 \\
$E-A A A / M-C T C$ & 11.5 & 20.2 & 22.2 & 78.0 \\
$E-A A G / M-A C G$ & 16.9 & 29.6 & 18.8 & 78.8 \\
$E-A G T / M-G C G$ & 13.7 & 24.1 & 58.2 & \\
Total & 42.2 & 73.9 & & \\
\hline
\end{tabular}

Genetic distances between pairs of clones were varied from 0.0093 to 0.1826 . No significant correlation between matrices of genetic and spatial distance between clones was detected $(r$ $=0.07)$. The number of clones detected $(G)$ divided by sample size $(N)$ was 0.216 , the estimate of Simpson's index of diversity $(D)$ was 0.4335 , and the genotypic richness, $\mathrm{R}=$ 0.2174 . The spatial distribution of the 35 clones in the plots is shown in Fig. 2. Clone 1 occupied a large area of both sites, but most of the sample clones were scattered irregularly and did not form groups (Fig. 2).
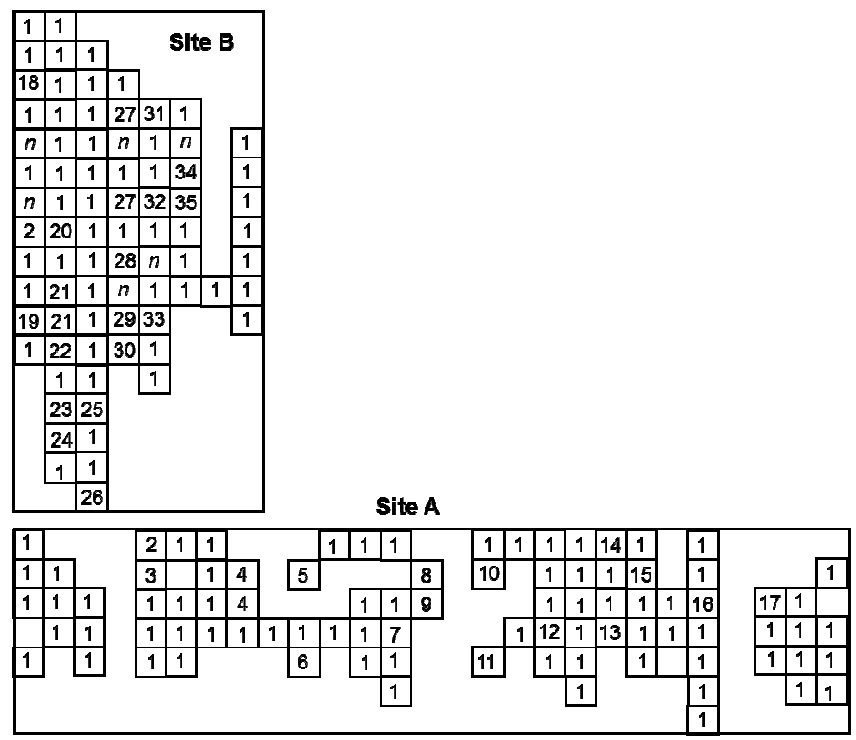

Figure 2. Spatial distribution of the clones of Nelumbo nucifera in the Lake Kasumigaura plots shown in Fig 1. Identical numbers indicate sampling sites of clones with identical AFLP band patterns. $n$ indicates no data.

\subsection{Uchi-numa Pond}

Out of 214 samples taken from Uchi-numa Pond, only two leaves in site B had an identical AFLP band pattern; the other 212 leaves showed unique patterns. In total, 213 AFLP band patterns were detected in the 214 samples, indicating that this population consisted of 213 clones. When the AFLP band patterns of the 213 clones were compared in pairs, total of 73.9 fragments could be detected using the 3 primer sets, and a mean of 58.2 fragments $(78.8 \%)$ were polymorphic between the clones in each pair (Table 1). The estimate of Simpson's index of diversity $(D)$ was 0 , and the genotypic richness, $\mathrm{R}=$ 0.9953 suggesting that genetic diversity for the population of Uchi-numa Pond was higher compare with the population of Lake Kasumigaura.

A genetic distance matrix was constructed with 40 clones selected from the central locations of each of the sites A and B were used to construct dendorogram using UPGMA. The cluster analysis generated trees with 6 clusters at site A and 5 clusters at site B (Upper panel in Fig. 3). The spatial distributions of the samples are shown in lower panel of Fig. 3. A matrix of the genetic distances among the samples from each site was correlated significantly with the corresponding matrix of spatial distances. The value of correlation coefficient (r) ranged from 0.1676 to $0.6636(\mathrm{P}<0.05)$ for the number of samples ( $\mathrm{n}$ ) ranging from 10 to 703 , indicating that the genetic distance between samples could be explained by the spatial distance. Samples included in the same cluster in Fig. 3 were usually distributed together along the margin of the pond, suggesting that seed dispersal occur along the flow of water. 
Site A

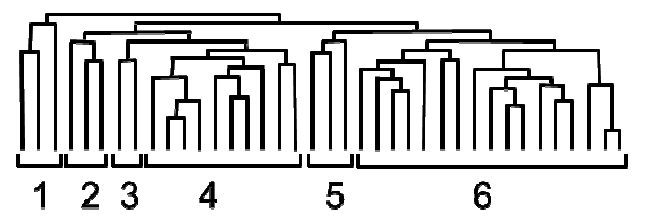

Site B

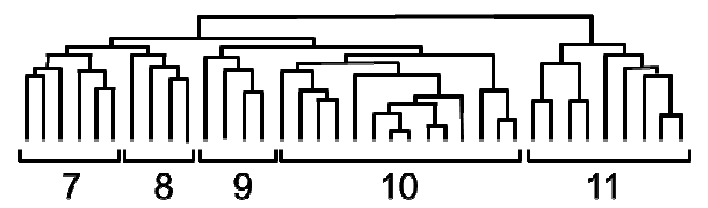

\begin{tabular}{|r|r|r|r|r|r|r|r|r|r|}
\hline 11 & $n$ & 11 & $\mathrm{n}$ & 11 & $n$ & 11 & 11 & 11 & 10 \\
\hline 11 & 10 & 10 & 10 & 8 & 10 & 10 & 10 & 10 & 10 \\
\hline 10 & 12 & 10 & 10 & 10 & 9 & 9 & 9 & 9 & 11 \\
\hline 7 & 7 & 7 & 7 & 7 & 7 & 8 & 8 & 8 & 10 \\
\hline
\end{tabular}

$\overline{10 \mathrm{~m}}$

Figure 3. UPGMA clustering (upper panel) based on pairwise distance between multilocus AFLP genotypes from Nelumbo nucifera samples and spatial distribution (lower panel) in the central areas of sites $A$ and B in Uchi-numa Pond. The numbers in lower panel indicate the cluster in the UPGMA tree in upper panel. $n$ indicates no data.

\section{Discussion}

The difference in genotypic diversity between the $N$. nucifera populations of Lake Kasumigaura and Uchi-numa Pond corresponds to a marked difference in the mode of reproduction. The spatial pattern of clones indicates that a large clone predominates in the plot area of Lake Kasumigaura and the particular single clone included 122 samples. These results suggest that the genetic diversity of the $N$. nucifera population in Lake Kasumigaura is low as a result of the wide distribution of a particular clone. On the other hand, many small patches of clones grow in Uchi-numa Pond indicating limited clonal reproduction. One of the possible reasons for the low level of genetic diversity in Lake Kasumigaura is that efficient sexual recruitment has been inhibited because of the superior competitive ability of rhizomes over seedlings. Growth of young seedlings derived from seeds is suppressed by surrounding large clones. In mature populations such as the Lake Kasumigaura population, seedling recruitment may be poor, resulting in populations consisting of small numbers of large genets. Our results suggest that clonal reproduction is dominance in stable environments such as Lake Kasumigaura, whereas seedling recruitment is dominance in unstable environments such as Uchi-numa Pond.

In $N$. nucifera and other species with both asexual and sexual reproduction, seeds are clearly the primary means of long-distance dispersal and are considered to be a prerequisite for persistence [21, 22]. Xue et al. [8] collected 47 accessions of wild lotus in Heilongjiang Province in China and examined genetic diversity using RAPD and ISSR angmarkers. Analysis of the percentages of polymorphic loci and expected heterozygosity, molecular variance and variance components showed that a small amount of variability exists among the populations in the river valleys. The very limited genetic diversity in these populations suggested that wild lotus in these locations has experienced a severe bottleneck, founder effect and rebirth effect. We found in this study that clonal propagation is more favoured in late successional stage like in Lake Kasumigaura and in relatively homogeneous habitat. Han et al. [6] and Yang et al (2012) [23] suggested that most of the genetic diversity was attributed to geographical isolation instead of their habitats because low sexual recruitment and clonal growth greatly reduced the diversity within the populations. They suggested that the low levels of genetic diversity harbored within their study populations may be due to the unique fruit structures, low inherent variability of the ancestral species, the repeated decrease and increase of temperature and the destructive utilization.

As illustrated by our results in lotus, the mode of reproduction has important effects on the spatial distribution of genetic variation within and among plant populations [18, 19, 24]. The trade-off between sexual and asexual reproduction of Scirpus mariqueter has been demonstrated in many species to be influenced by plant size, population density, resource level and age [25]. Newell and Tramer [26] predicted that seed production should decline with increasing competition in herbaceous plants. Loehle (1987) [27] suggested that sexual reproduction in clonal plants is favored by low density or favorable site conditions.

A hypothetical description of development of clonal plant population by Kays and Harper [28], flux of vegetatively propagated ramets eventually dominates the dynamics of the developing local population. However, this is not true for all clonal plants [5]. Hartnett and Bazzaz [29] described that new genets of the clonal plant Solidago canadensis (Canada goldenrod) were recruited only during a limited period and the number of genets slowly declined and seemed to reach equilibrium in older population after an early maximum in genet density. Barrett and Silander [30] reported that new genets of white clover (Trifolium repens) were recruited into the population each year. Even though disturbances favor germination, survivorship of seedlings was higher in undisturbed vegetation. In contrast to the situation in Solidago canadensis, intraspecific competition in $T$. repens does not completely suppress recruitment. Jacquemyn et al. [23] showed that sexual reproduction of Paris quadrifolia was primarily found in moist and relatively productive sites, whereas under stressful conditions sexual reproduction and seedling recruitment were suppressed. Studies by Zhang and Yong [31] of ramet dynamics in Scirpus mariqueter have 
revealed that, in general, no plants that originate from seeds survive in mature $S$. mariqueter communities, and that the initial colonization on bare beaches often begins from vegetative parts that have been transported from high or medium elevations. Araki and Kadono [9] studied the reproductive contribution of seeds in normal pollen-producing populations of Utricularia australis; their results were consistent with a model of clonal dominance and rarity of seed or seedling survival in natural populations. From our results in $N$. nucifera, it was suggested that seedling recruitment are favored at low density without competition with large clones, whereas seedling growth is limited in mature population. Sexual reproduction produces new genets and younger population exhibit higher genetic diversity.

Eckert et al. [32] investigated whether differences in reproductive strategy influence the diversity of genotypes within populations of diploid and triploid plants of Butomus umbellatus. Contrary to expectations, sexually fertile populations did not exhibit higher genotypic diversity than sterile populations, suggesting that the potential for recombination in diploids is not realized because seeds are outcompeted by bulbils during establishment.

It was found that clonal reproduction does not bear genetic diversity in mature population like in Lake Kasumigaura, however, the population with sexual reproduction contains the potentiality to produce genetic diversity by seedling recruitment. In newly established population with high genetic diversity like in Uchi-numa Pond, clones closest locations to one another are still likely to be genetically more related. Almost every sample from Uchi-numa Pond displayed a different genotype, but genetically related samples were located within a short distance from one another along the margin of the pond. It can be assumed that the seeds derived from particular mother plants spread along with a generous flow of the pond and germinate. Identification of mother plants should be required by genomic analyses of mitochondria or/and chloroplasts.

\section{Conclusions}

Clonal species can produce offspring through clonal propagation and sexual reproduction. The mode of reproduction, sexual vs. asexual, is likely to have important effects on the spatial distribution of genetic variation within and among plant populations. Genetic diversity of 2 wild lotus populations in Japan different in the environment was estimated by an AFLP fingerprinting. Our results presented that the spatial pattern of clones indicates several large clones distributed in mature population like Lake Kasumigaura and many small patches of clones grow in immature population like Uchinuma Pond. Asexual reproduction is relatively dominance in stable environment, whereas sexual reproduction is favored when the water level varies recurrently to damage the population, because small seedlings derived from seeds could not survive in mature $N$. nucifera population. Populations with the capacity for sexual reproduction will contain more genotypic diversity.

\section{Acknowledgements}

We thank Dr. Hiroshi Ikehashi, Nihon University for the helpful discussion and advice, and Minami Shibata, Kentaro Tajima, and Hiroyuki Hayashi, Nihon University for sampling and technical assistance.

\section{References}

[1] Kunii, H. and M. Maeda 1982. Seasonal and long-term changes in surface cover of aquatic plants in a shallow pond, Ojaga-ike, Chiba, Japan. Hydrobiologia 87: 45-55.

[2] Sastroutomo, S.S. 1982. Summer biomass of aquatic macrophytes in relation to sediment characteristics in Lake Aino-mura, Miyagi. Japan. J. Ecol. 32: 45-55.

[3] Nohara, S. and M. Kimura 1997. Growth characteristics of Nelumbo nucifera Gaertn. In response to water depth and flooding. Ecol. Res. 12: 11-20.

[4] Carpenter, S.R. and Lodge, D.M. 1986. Effects of submersed macrophytes on ecosystem processes. Aqu. Bot. 26: 341-370.

[5] Eriksson, O. 1993. Dynamics of genets of clonal plants. Trend. Ecol. Evo. 8: 313-316.

[6] Han, Y.C., Teng, C.Z., Wahiti, G.R., Zhou, M.Q., Hu ZL and Y.C. Song 2009. Mating system and genetic diversity in natural populations of Nelumbo nucifera (Nelumbonaceae) detected by ISSR markers. Plant Syst. Evo. 277: 13-20.

[7] Katori, M., K. Nomura and K. Yoneda. 2002. Propagation of flowering lotus (Nelumbo nucifera Gaertn.) by rhizome straps, without enlarged rhizomes. Jpn. J. Trop. Agr. 46: 195-197.

[8] Xue, J., Zhuo, L. and S. Zhou 2006. Genetic diversity and geographic pattern of wild lotus (Nelunbo nucifera) in Heilongjiang Province. Chin. Sci. Bul. 51: 421-432.

[9] Araki, S. and Kadono, Y. 2003. Restricted seed contribution and clonal dominance in a free-floating aquatic plant Utricularia australis R.Br. in southwestern Japan. Ecological Research. 18: 599-609.

[10] Barrett, S.C.H., Eckert, C.G. and Husbund, B. C. 1993. Evolutionary processes in aquatic plant populations. Aquatic Botany. 44: 105-145.

[11] Kameyama, Y. and M. Ohara 2006. Genetic structure in aquatic bladderworts: Clonal propagation and hybrid perpetuation. Ann. Bot. 98: 1017-1024.

[12] Pollux, B.J.A., M.D.E.Jong, A. Steegh, E. Verbruggen, and J.M.Van Groenendael 2007. Reproductive strategy, clonal structure and genetic diversity in populations of the aquatic macrophyte Sparganium. Mol. Ecol. 16: 313-325.

[13] Nohara, S. and T. Tsuchiya 1990. Effects of water level fluctuation on the growth of Nelumbo nucifera Gaertn. in Kasumigaura, Japan. Ecol. Res. 5: 237-252.

[14] Murray, M.G. and W.F. Thompson 1980. Rapid isolation of high molecular weight plant DNA. Nucleic Acids Res. 8: 4321-4325. 
[15] Vos, P., Hogers, R., Bleeker, M., Reijans, M., Lee, T. van de, Hornes, M., Frijters, A., Pot, J., Peleman, J. Kuiper, M. and M. Zabeau 1995. AFLP: a new technique for DNA fingerprinting. Nucleic Acids Res. 23: 4407-4414.

[16] Nei, M. and W.H. Li 1979. Mathematical model for studying genetic variation in terms of restriction endonucleases. Proc. Natl. Acad. Sci. USA 76: 5269-5273.

[17] Felsenstein, J. 2005. PHYLIP (Phylogeny Inference Package) version 3.6. Distributed by the author. Department of Genome Sciences, University of Washington, Seattle.

[18] Ellstrand N.C. and M.L. Roose 1987. Patterns of genotypic diversity in clonal plant species. Amer. J. Bot. 74: 123-131.

[19] Widén, B.S., Cronberg, N. and M. Widén 1994. Genotypic diversity, molecular markers and spatial distribution of genets in clonal plants. A literature survey. Folia Geobot. Phytotax. 29: 245-263.

[20] Doken, M.E. and Eckert, C.G. 2001. Severely reduced sexual reproduction in northern populations of a clonal plant, Decodon verticillatus (Lythraceae). J. Ecol. 89: 339-350.

[21] Olivieri, I., Michalaskis, Y. and P.H. Gouyon 1995. Metapopulation genetics and the evolution of dispersal. Amer. Nat. 146: 202-228.

[22] Husband, B.C. and S.C.H. Barrett 1996. A metapopulation perspective in plant population biology. J. Ecol. 84: 461-469.

[23] Yang, M., Y. Han, L. Xu, J. Zhao and Y. Liu 2012. Comparative analysis og genetic diversity of lotus (Nelumbo) using SSR and SRAP markers. Scientia Horticulturae 142: 185-195.
[24] Jacquemyn, H., Brys, R., Honnay, O., Hermy, M. and I. Roldan-Ruiz 2005. Local forest environment largely affects below-ground growth, clonal diversity and fine-scale spatial genetic structure in the temperate deciduous forest herb Paris quadrifolia. Mol. Ecol. 14: 4479-4488.

[25] Sun, S., Gao, X. and Y. Cai 2001. Variations in sexual and asexual reproduction of Scirpus mariqueter along an elevational gradient. Ecol. Res. 16: 263-274.

[26] Newell, S.J. and E.J. Tramer 1978. Reproductive strategies in herbaceous plant communities during succession. Ecology 59: 228-234.

[27] Loehle, C. 1987. Partitioning of reproductive effort in clonal plants: a benefit-cost model. Oikos 49: 199-208.

[28] Kays, S. and J.L. Harper 1974. The regulation of plant and tiller density in a grass sward. J. Ecol. 62: 97-105.

[29] Hartnett, D.C. and F.A. Bazzaz 1985. The genet and ramet population dynamics of Solidago canadensis in an abandoned field. J. Ecol. 73: 407-413.

[30] Barrett, J.P. and Silander, J.A. 1992. Seedling recruitment limitation in white clover (Trifolium repens; Leguminoseae). Amer. J. Bot. 79: 643-649.

[31] Zhang, L.Q. and X.K. Yong 1992. Dynamics of ramet density and biomass in Scirpus mariqueter. Acta Phytoecol. Sinica 16: 315-325.

[32] Eckert, C.G., Lui, K., Bronson, K., Corradini, P. and Bruneau, A. 2003. Population genetic consequences of extreme variation in sexual and clonal reproduction in an aquatic plant. Mol. Ecol. 12: 331-344. 